\begin{tabular}{c|c|c|}
\cline { 2 - 3 } CRITICAL & $\begin{array}{l}\text { Western Ghats } \\
\text { EARTNERSHIP FUND }\end{array}$ & $\begin{array}{l}\text { Special Series } \\
\text { PAR Secial }\end{array}$ \\
\hline
\end{tabular}

\title{
Diversity of rhacophorids (Amphibia: Anura) in Parambikulam Tiger Reserve, Western Ghats, Kerala, India
}

\author{
K.M. Jobin ${ }^{1}$ \& P.O. Nameer ${ }^{2}$ \\ 1,2 Department of Wildlife Sciences, College of Forestry, Kerala Agricultural University (KAU), Vellanikkara, Kerala 680656, India \\ Email: ${ }^{1}$ jobinmathew00@gmail.com, ${ }^{2}$ nameerpo@gmail.com (corresponding author)
}

Abstract: A study on the rhacophorids of Parambikulam Tiger Reserve was conducted from April to July 2011. Eleven species of rhacophorids in four genera-Rhacophorus (three species), Polypedates (one species), Pseudophilautus (one species) and Raorchestes (six species) - were recorded. Distribution, natural history and biological information is provided including some recommendations for changes in the IUCN conservation status of the rhacophorids of Western Ghats have been proposed in the paper.

Keywords: IUCN Red Listing, Kerala, Parambikulam Tiger Reserve, Rhacophoridae, Western Ghats.

\section{Date of publication (online): 26 October 2012}

Date of publication (print): 26 October 2012

ISSN 0974-7907 (online) | 0974-7893 (print)

Editor: Sanjay Molur

\section{Manuscript details:}

Ms \# 03081

Received 25 January 2012

Final received 28 July 2012

Finally accepted 14 August 2012

Citation: Jobin, K.M. \& P.O. Nameer (2012). Diversity of rhacophorids (Amphibia: Anura) in Parambikulam Tiger Reserve, Western Ghats, Kerala, India. Journal of Threatened Taxa 4(13): 3205-3214.

Copyright: (c) K.M. Jobin \& P.O. Nameer 2012. Creative Commons Attribution 3.0 Unported License. JoTT allows unrestricted use of this article in any medium for non-profit purposes, reproduction and distribution by providing adequate credit to the authors and the source of publication.

Acknowledgements: We would like to thank the Kerala State Forest Department, particularly the Chief Wildlife Warden for granting permission (study permit No. WL 12-7972/2010) to study the amphibians at PKTR We thank the Field Director, PKTR, the Wildlife Warden, the Assistan Wildlife Wardens, the Wildlife Asst. and other staff of PTR for making necessary arrangements on the logistics at PTR. We also thank Dr. Anil Zachariah and Sandeep Das for confirming the identification of the frogs, Mr. Sreenivasan, the forest watcher at the PKTR for accompanying us in the field, Sreehari R., Kiran Thomas, Maya T. Joy, Lakshmi, A. Nithin S. Sachin, K. Aravind for their support, V.S. Sreehari for helping with the preparation of the map of PKTR. The Associate Dean of the College of Forestry is thanked for the encouragement and the facilities during the study. We also would like to thank two anonymous reviewers for offering very valuable comments.

ZooBank urn:Isid:zoobank.org:pub:450A0968-536A-4E4A-9F60AF0DB8415D01

\section{OPEN ACGESS | FREE DOWNLOAD CC (1) Cு}

Western Ghats, one of the 34 biodiversity hotspots of the World (Myers et al. 2000; Conservation International 2005) is exceptionally rich in amphibian diversity. During the last one and half decades there has been an outburst of publications, including the description of several new species to science from the family Rhacophoridae (Das \& Ravichandran 1998; Vasudevan \& Dutta 2000; Bossuyt 2002; Kuramoto \& Joshy 2003; Biju \& Bossuyt 2005a,b; Biju \& Bossuyt 2006a; Das \& Dutta 2006; Gururaja et al. 2007; Biju \& Bossuyt 2009; Biju etal. 2010; Zachariah etal. 2011a,b). About 68 species of frogs were described from India in the last one decade of which 32 species, nearly $50 \%$, were members of the family Rhacophoridae (Dinesh et al. 2011). The rhacophorids are characterised by their varied microhabitat preferences ranging from ground litter (Biju et al. 2010), bushes and reeds (Gururaja et al. 2007) to high up in the forest canopy (Biju \& Bossuyt 2005a). The rhacophorids also display varying breeding biology (Patil \& Kanamadi 1997; Krishnamurthy et al. 2002; Biju 2003; Gururaja \& Ramachandra 2006). Very little is known about the amphibians of Parambikulam Tiger Reserve (PKTR) in general and rhacophorids in particular hence the present study. The earlier studies on the amphibians of PKTR include Rao (1937), Satyamurthi (1967) and Radhakrishnan (1996).

The publication of this article is supported by the Critical Ecosystem Partnership Fund (CEPF) -- a joint initiative of l'Agence Française de Développement, Conservation International, the Global Environment Facility, the Government of Japan, the MacArthur Foundation and the World Bank. 


\section{Study Area}

Parambikulam Tiger Reserve (PKTR) is situated in Palghat District, Kerala, India, within the Anamalai Hills and borders Nelliyampathy Hills (76035'$\left.76^{0} 50^{\prime} \mathrm{E} \& 1^{0} 20^{\prime}-10^{\circ} 26^{\prime} \mathrm{N}\right)$. The total extent of the Tiger Reserve is $643.66 \mathrm{~km}^{2}$, with a core zone of $390.89 \mathrm{~km}^{2}$ and buffer zone of $252.77 \mathrm{~km}^{2}$ (Kaler 2011 ). The major vegetation types in the core zone are IA/ $\mathrm{C} 4$ west coast tropical evergreen forests, $2 \mathrm{~A} / \mathrm{C} 2$ west coast tropical semievergreen forests, $3 \mathrm{~B} / \mathrm{C} 2$ southern moist mixed deciduous forests, $5 \mathrm{~A} / \mathrm{C} 3$ southern dry mixed deciduous forests, 2/E3 moist bamboo brakes, $8 \mathrm{~A} / \mathrm{C} 1 / \mathrm{E} 1$ reed brakes and IIA/C1 southern montane wet temperate forests (sholas). The altitude of the PKTR ranges from 300-1438 m (Kaler 2011). Major peaks are Karimala (1438m), Pandaravarai (1290m), Vengoli (1120m) and Puliyarapadam (1010m). PKTR has three man-made reservoirs namely Parambikulam, Thunacadavu and Peruvaripallam whose cumulative waterspread area is $20.66 \mathrm{~km}^{2}$. The PKTR forms the catchment of Chalakkudy River. Administratively, PKTR is divided into four ranges, the Karimala Range, Orukomban Range, Parambikulam Range and Sungam Range (Fig. 1). The present study is primarily confined to the core zone of PKTR.

\section{Methods}

Visual encounter surveys were carried out at Parambikulam TR from April-July 2011. Efforts were made to cover all the representative habitats of PKTR. The frogs were searched for in the suitable microhabitats and two specimens per species were collected. Standard morphological measurements were taken for all specimens as per Bossuyt \& Dubois (2001). These include, snout vent length (SVL), head width (HW), head length (HL), maximum distance between upper eye lid (IUE), maximum width of upper eye lid (UEW), snout length (SL), eye length (EL), forelimb length (FLL), hand length (HAL), thigh length (TL), shank length (SHL), tarsus length (TaL), foot length (FoL). The measurements were taken using a Mitutoyo Digimatic Caliper (to the nearest $0.1 \mathrm{~mm})$. The collected specimens were fixed using $5 \%$ formaldehyde, preserved in $70 \%$ ethyl alcohol and were deposited in the Kerala Agricultural University Natural History Museum (KAUNHM), Thrissur with appropriate registration numbers. In this paper the rhacophorid frogs obtained from PKTR were grouped into "large frogs" (SVL >50mm), "medium frogs" (SVL 30-50mm) and "small frogs" (SVL < 30mm) based on the snout-vent length (SVL). The frogs were identified by comparing the standard measurements

76035'0"E

76040'0”'E $76^{\circ} 50^{\prime} 0^{\prime \prime}$

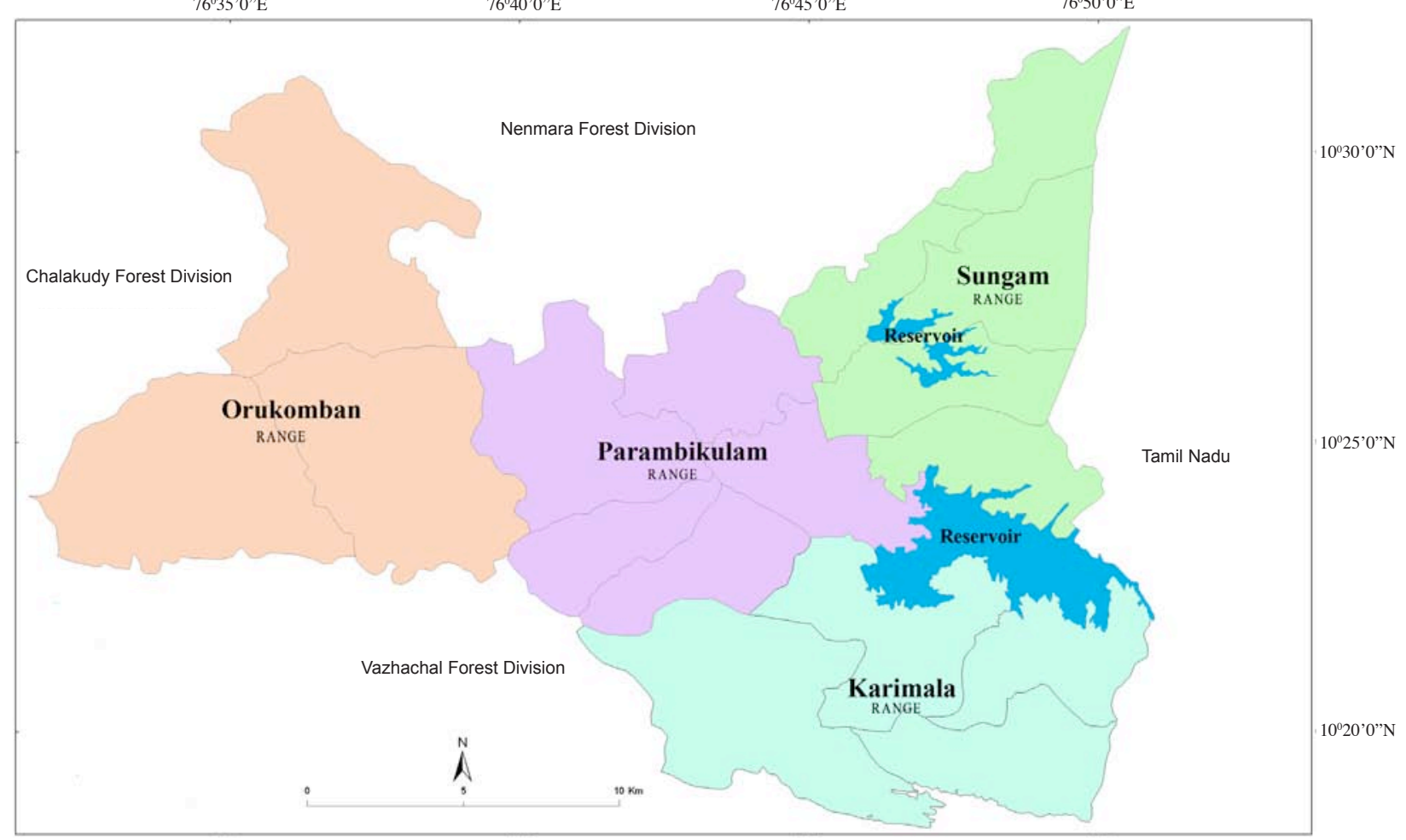

Figure 1. Map of Parambikulam Tiger Reserve 
taken on the frogs with the published literature.

\section{Results}

Eleven species of rhacophorid frogs in four genera, namely, Rhacophorus (three species), Polypedates (one species), Pseudophilautus (one species) and Raorchestes (six species) were recorded from PKTR during the present study. All the 11 species of rhacophorids recorded from PKTR are endemic to the Western Ghats.

1. Charpa Tree Frog Polypedates occidentalis Das \& Dutta, 2006

A large frog $(\mathrm{SVL}=55 \mathrm{~mm})$ having dark yellowishbrown color with a dark hour-glass-shaped mark on dorsum. Distinct marbling on the sides of thigh and belly. Snout pointed with nostrils closer to snout tip. Supra tympanic fold prominent, fingers not webbed (Image 1).

One individual was encountered on a wet rock near a stream flowing through a reed (Ochlandra sp.) patch at Malakkapara, buffer zone of PKTR. This location is about $50 \mathrm{~km}$ north to the type locality of this species (Charpa in Vazhachal Forest Division). Polypedates occidentalis is also reported from Karnataka (Hegde \& Bhat 2011). It is considered a 'Data Deficient' species, according to IUCN (Das 2008) calling for a reassessment based on new information available for the species, which is probably adequate with respect to the distribution range and threats.

2. Kani Bush Frog Pseudophilautus kani (Biju \& Bossuyt, 2009)

A small frog $(\mathrm{SVL}=27.52+1.71 \mathrm{~mm}$, Table 1$)$ with brown to light grayish-brown dorsum. Shank length almost equal to the thigh length. Canthus rostralis and loreal region dark grayish-brown, prominent supra tympanic fold, $2 / 3^{\text {rd }}$ of tympanum including supra tympanic fold blackish (Image 2). Hind limbs possess rudimentary webbing while webbing is lacking in the forelimbs.

Calling males were encountered from bushes and female frogs from ground litter. In PKTR, they are seen from 400-1400 $\mathrm{m}$ at the top of Karimalagopuram. P. kani has been sighted from teak plantations, moist deciduous forests and evergreen forests and is one of the commonest bush frogs in PKTR.

Biju \& Bossuyt (2009) had reported P. kani only from the Agasthyamala region, south of the Shengotta Gap in Western Ghats. Thus, the present sighting of

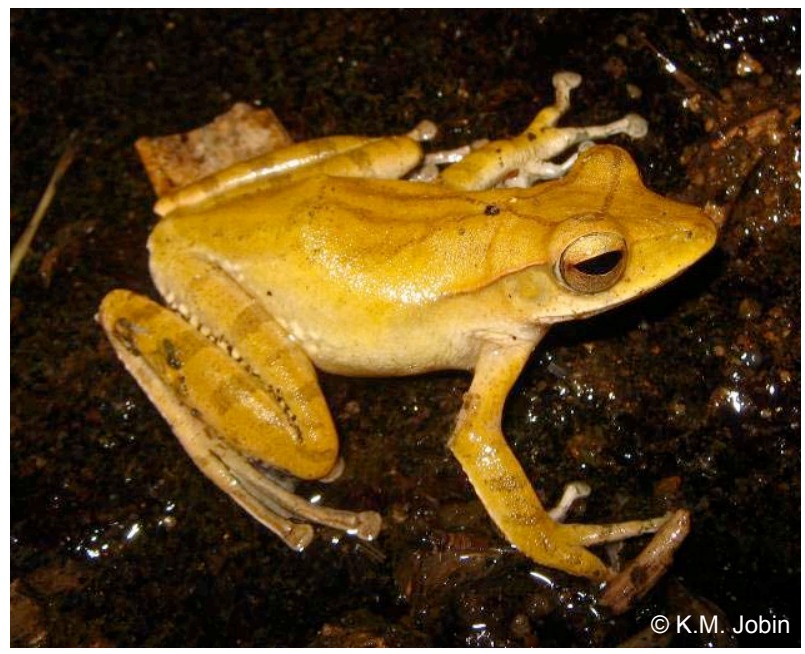

Image 1. Polypedates occidentalis Das \& Dutta, 2006

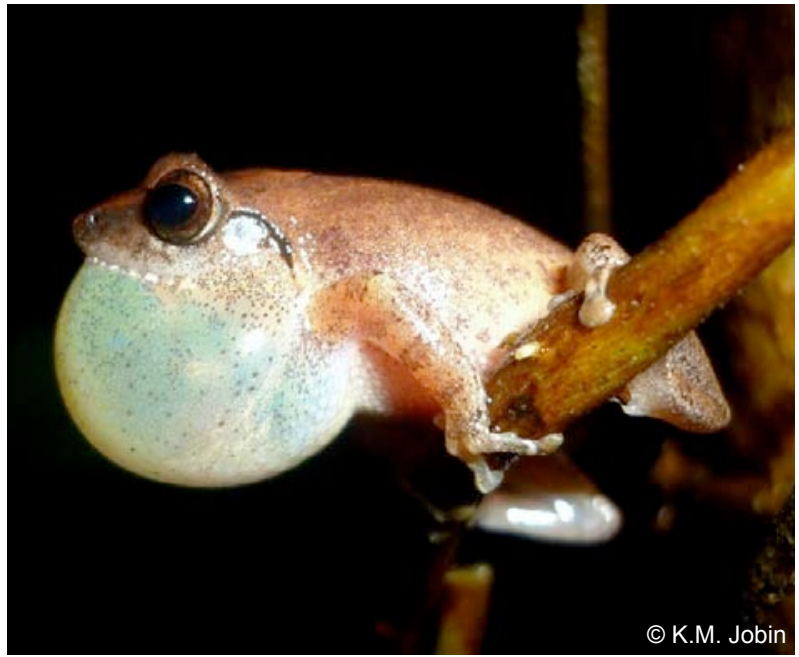

Image 2. Pseudophilautus kani (Biju \& Bossuyt, 2009)

this species from the Anamalai region is of interest. The SVL of the female P. kani, reported by Biju \& Bossuyt (2009) was only $24.4 \mathrm{~mm}$, while the average SVL measurement for the females that we got from PKTR measured $27.52 \mathrm{~mm}$.

\section{Variable Bush Frog Raorchestes akroparallagi} (Biju \& Bossuyt, 2009)

A small frog $(\mathrm{SVL}=21.46+0.46 \mathrm{~mm}$, Table 1) with a bright light green dorsum. The snout pointed, canthus rostralis, loreal, supra tympanic fold and forearm creamy to pale brown with numerous dark brown spots (Image 3).

At, PKTR it was encountered throughout from an altitude of $450-1000 \mathrm{~m}$ and above, from the teak plantations, moist deciduous forests and evergreen 
forests. The locations from where this frog was sighted include the Parambikulam earth dam, base of Karimalagopuram, Kuriyarkutti, Orukomban in PKTR and from Thoothanpaara Estate in Nelliampathies in the buffer region. Biju \& Bossuyt (2009) reported this species from Wayanad and Agasthyamala regions of Western Ghats in Kerala. This is the first report of this species from the Anamalais of the Western Ghats. There is adequate information from published sources to assess this species which has not been evaluated until now.

4. Anil's Bush Frog Raorchestes anili (Biju \& Bossuyt, 2006)

A small bush frog $(\mathrm{SVL}=24.81+0.51 \mathrm{~mm}$, Table 1), narrow pointed snout, head width which is more than head length. Light chocolate brown color with a dark inverted 'V' pattern on dorsum. Supra tympanic fold prominent, canthus rostralis, loreal and supra tympanic fold dark brown. Metallic silver colored iris. Blackish blotches on lateral sides of abdomen, dark chocolate brown bands on both forelimbs and hind limbs (Image 4).

At PKTR $R$. anili was encountered at Poopara region in the core zone and from Malakkapara in the buffer zone. At Poopara the calling males were found primarily on the leaves of Curcuma sp. leaves near an evergreen patch at an altitude of $840 \mathrm{~m}$. While at Malakkapara $R$. anili was found among the pebbles and leaf litter in a stream. Biju \& Bossuyt (2009) reported this species from Wayanad and Agasthyamala regions of Western Ghats in Kerala. The present sighting thus is the first report of this species from the Anamalais of the Western Ghats.

According to IUCN red listing it is a 'Least Concern' species (Biju 2006). Taking into account the fact that $R$. anili is endemic to the Western Ghats, known only from four locations within southern Western Ghats, of which two sites are located in nonprotected areas. And also the fact that the nonprotected areas within the Western Ghats are facing extreme stress from various forms of anthropogenic factors, which in turn may adversely affect the quality of the microhabitat parameters for the survival of amphibians, a is reassessment recommended for this species.

5. Jayaram's Bush Frog Raorchestes jayarami (Biju \& Bossuyt, 2009)

Small sized bush frog $(\mathrm{SVL}=22.17+0.79 \mathrm{~mm}$, Table 1) with a bright green dorsum. Green coloration

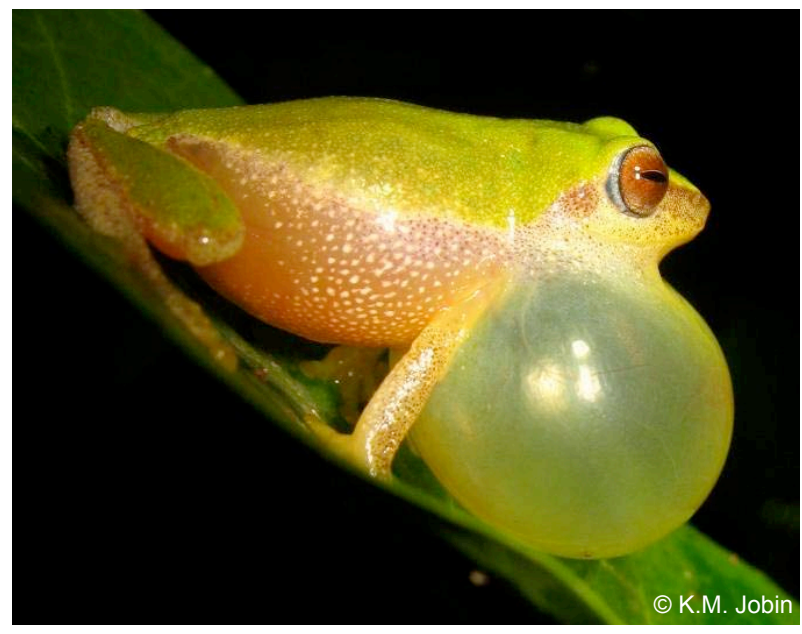

Image 3. Raorchestes akroparallagi (Biju \& Bossuyt, 2009)

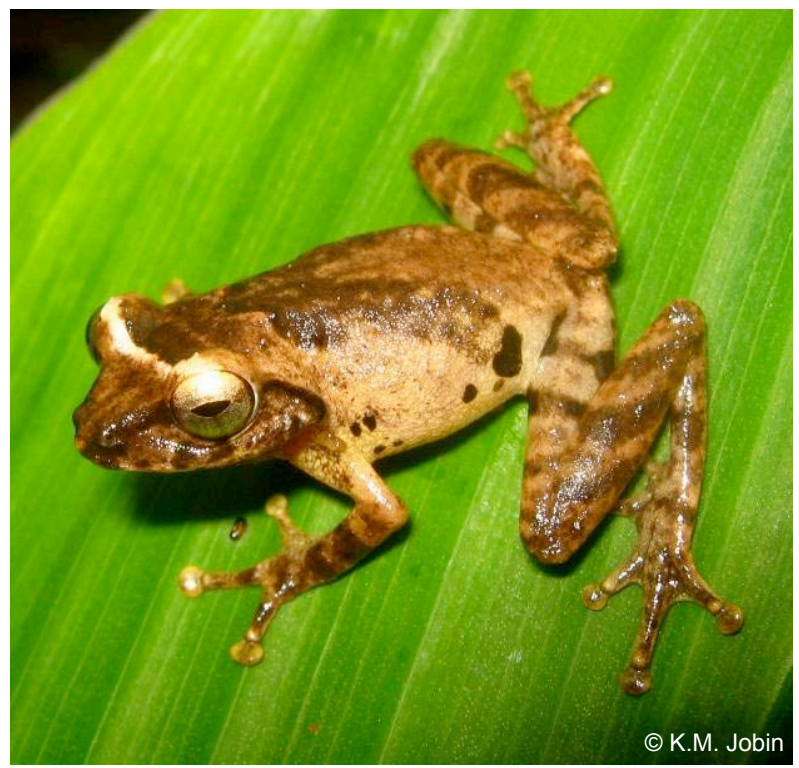

Image 4. Raorchestes anili (Biju \& Bossuyt, 2006)

extending to the lower arm of forelimb leaving the upper arm white. Snout pointed, tympanum and supra tympanic fold not distinct. Lateral part of abdomen and limbs white or rarely with a sky blue tinge (Image $5)$.

At PKTR, $R$. jayarami was sighted only above $1000 \mathrm{~m}$ altitude in the evergreen patches of Karimalagopuram and several calling male frogs were also sighted in the tea plantation near the evergreen patch at Shekkalmudi. Biju \& Bossuyt (2009) reported this species from Valparai within the Anamalai Hills of the southern Western Ghats in Tamil Nadu. Thus, the present sighting from the Kerala part of the Anamalai Hills is of interest. 


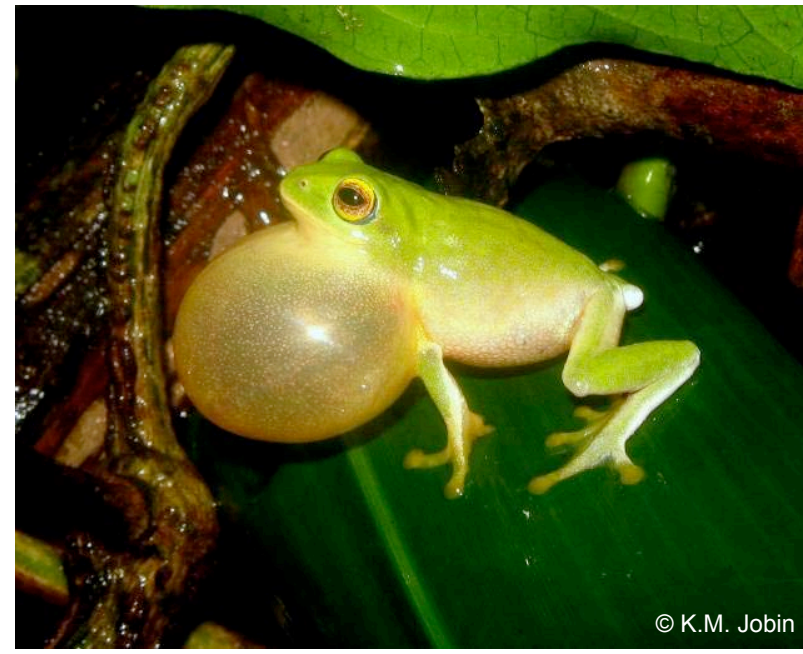

Image 5. Raorchestes jayarami (Biju \& Bossuyt, 2009)

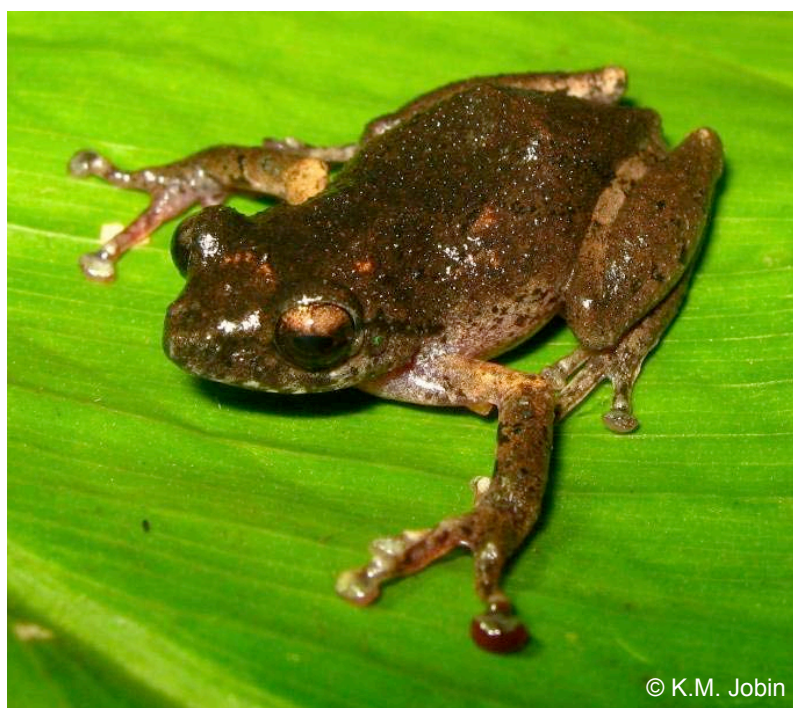

Image 6. Raorchestes marki (Biju \& Bossuyt, 2009)
This species is not evaluated and needs assessment by the IUCN Red List.

6. Mark's Bush Frog Raorchestes marki (Biju \& Bossuyt, 2009)

Small sized bush frog $(\mathrm{SVL}=20.71+0.06 \mathrm{~mm}$, Table 1) with dark chocolate brown to dark brown color with reddish markings on the body, pointed snout, shank longer than the thigh. Eyes protruding with coppery iris. Supra tympanic fold prominent. Lateral sides of limbs and abdomen possess small blackish spots or blotches (Image 6).

$R$. marki were encountered from a semi-evergreen patch on top of Vengoli Hill, at an altitude of $1120 \mathrm{~m}$. Calling males were sighted among bushes and tree bark at a height of 1-2 m. The type locality of this species is Kaikatty in Nelliampathies (Biju \& Bossuyt 2009) and is known until now only from the type locality. The present observation from PKTR thus is a new site record for $R$. marki. However, both these locations are within the Anamalai Hills of the Western Ghats. According to the IUCN criteria $\mathrm{B} 1 \mathrm{ab}$ (iii) $+2 \mathrm{ab}$ (iii) ver 3.1, it is a 'Critically Endangered' species (IUCN SSC Amphibian Specialist Group, 2011).

7. Kalpetta Bush Frog Raorchestes nerostagona (Biju \& Bossuyt, 2005)

Medium sized $(\mathrm{SVL}=34 \mathrm{~mm}$ ) canopy bush frog with skin looking similar to bark of a tree. Toe webbed, dermal fringe present along outer margin of forelimb and hind limb. Prominent tympanum and supratympanic fold present. Shank longer than thigh and dark brown bands present. Male frogs will make a characteristic single noted call from the canopies during the breeding season.

Thisspecieswasidentified basedonthecharacteristic call of the frog, which was heard from the canopy of the evergreen forest patches in Shakkalmudi in PKTR and Thoothanpaara estate in the buffer region, between 800-1000 m. Biju \& Bossuyt (2009) have reported this species from Wayanad region of Western Ghats in Kerala. Purushotham \& Tapley (2011) have reported this species from Agumbe Rainforest Research Station, Agumbe in Karnataka. The present report from PKTR within the Anamalai Hills is thus the first record of this species from south of Palghat gap. According to the IUCN criteria B1ab (iii) ver 3.1, it is an Endangered species (Biju \& Bossuyt 2006b).

8. Ponmudi Bush Frog Raorchestes ponmudi (Biju $\&$ Bossuyt, 2005)

Medium sized frog $(\mathrm{SVL}=35.51+2.52 \mathrm{~mm}$, Table 1$)$ with robust body and protruding eyes. Light chocolate brown or creamy white color and rarely white spots on the dorsum. Snout round, toe partially webbed, lateral side of thigh and tarsus possess light grayish-white or orange brown marblings (Image 7).

Calling male frogs were sighted from semievergreen forests at Poopara and Vengoli in the core zone of PKTR and also seen from Malakkappara, in the buffer zone of PKTR at an altitude of $700 \mathrm{~m}$ and above. Biju \& Bossuyt (2009) have reported this species from Kalpetta, Mananthavady and Sulthan's Battery from Wayanad District, Gavi and Vagaman in 
Idukki District and Ponmudi in Thiruvananthapuram District of Kerala and Valparai in Tamil Nadu. Calling males were seen at a height ranging from 1-6 $\mathrm{m}$ from ground in PKTR. However, the individuals from the Malakkapara in the buffer zone of PKTR were sighted among decayed wood on the ground and another from a tree hole at a height of $1 \mathrm{~m}$ in an evergreen patch. Biju \& Bossuyt (2009) had sighted the species at a height of 8-15 $\mathrm{m}$ in Ponmudi and Valparai, while at Kalpetta it was sighted at a height of 2-4 m height. Purushotham \& Tapley (2011) reported this species from Agumbe Rainforest Research Station, Agumbe in Karnataka. The present sighting thus is the first report of this species from the Kerala part of the Anamalai Hills.

According to the IUCN criteria Blab(iii), it is a Critically Endangered species (Biju 2004). However, taking into account the present understanding on the distribution of the species from across Kerala, Tamil Nadu and Karnataka, a reassessment of the Raorchestes ponmudi would probably downlist the conservation status to Vulnerable.

9. Kalakkad Tree Frog Rhacophorus calcadensis Ahl, 1927

Medium sized $(\mathrm{SVL}=37.03+0.81 \mathrm{~mm}$, Table 1$)$ slender frog with dark brown to light reddish-brown color dorsum with darker patches and possess a tubercles on dorsal skin and on lower labials. Shank length almost similar to thigh length. Prominent dermal fringe along the outer margin of the forelimb and hind limb present. Dark bands in the forelimb and hindlimb (Image 8).

Four individuals were sighted among the bushes near a water hole between the evergreen forest and tea plantation at Shekkalmudi in PKTR at an altitude of $1118 \mathrm{~m}$. R. calcadensis is known only from KalakkadMundanthurai Tiger Reserve in Tamil Nadu (Ahl 1927; Vasudevan et al. 2001). The present record is thus the first report of this species from the Anamalai Hills of the Western Ghats.

According to the IUCN criteria Blab(iii), it is Endangered (Biju et al. 2004b). However, taking into account the present understanding on the distribution of the species, a reassessment of the Rhacophorus calcadensis could probably downlist the conservation status to Vulnerable based on the distribution range.

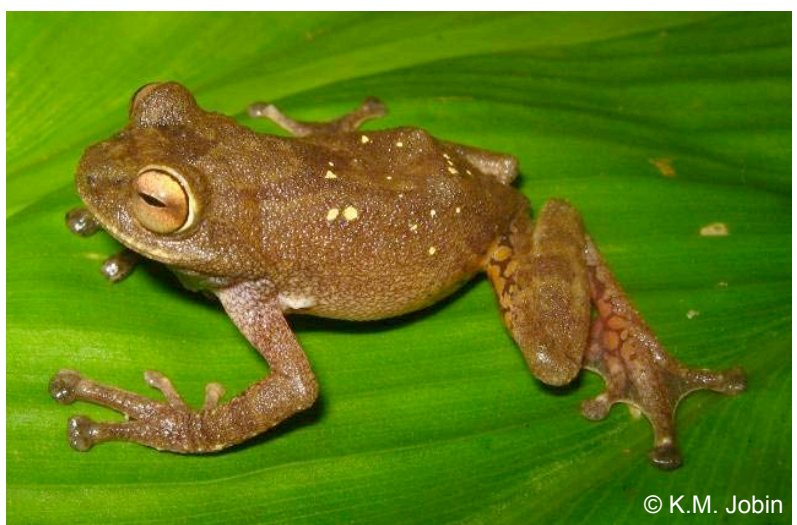

Image 7. Raorchestes ponmudi (Biju \& Bossuyt, 2005)

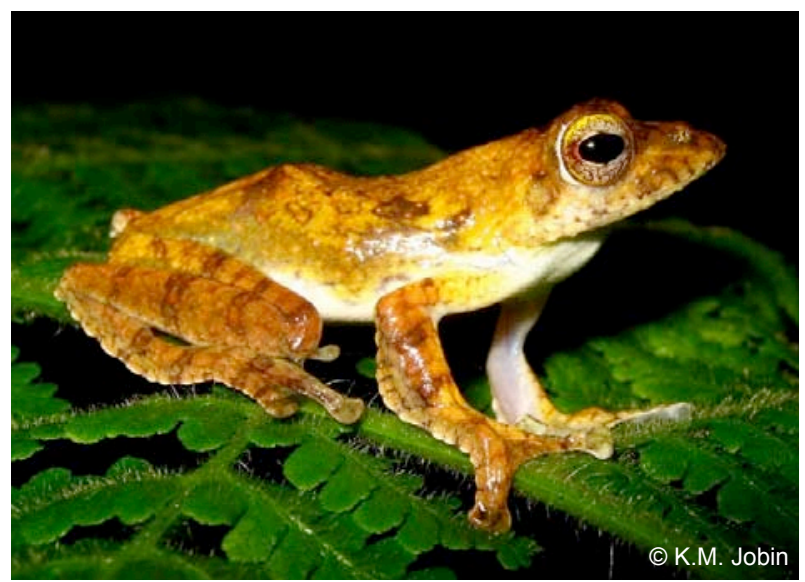

Image 8. Rhacophorus calcadensis Ahl, 1927

10. Malabar Gliding Frog Rhacophorus malabaricus Jerdon, 1870

A large sized tree frog $(\mathrm{SVL}=70 \mathrm{~mm}$, Table 1$)$ with a green dorsum and a prominent red colored web between fingers of hindlimb and forelimb. Head width larger than head length. Dermal fold along with the outer margin of the arm and on hindlimb from tarsus to tip of fourth toe (Image 9).

This species was located from Parambikulam earthdam and Kuriarkutti at an altitude of $565 \mathrm{~m}$ in PKTR. Rhacophorus malabaricus is a widely distributed species in Western Ghats from southern Maharashtra, Goa, Karnataka, Tamil Nadu and Kerala.

11. False Malabar Tree Frog Rhacophorus pseudomalabaricus Vasudevan \& Dutta, 2000

Medium sized (SVL 46.54+2.16 mm, Table 1) frog with green dorsum with dark stripes like venation of leaves on the green dorsum and on dorsal surface of limbs. Eyes are protruding, distinct supra-tympanic fold present. Dermal fold along the outer margin of 


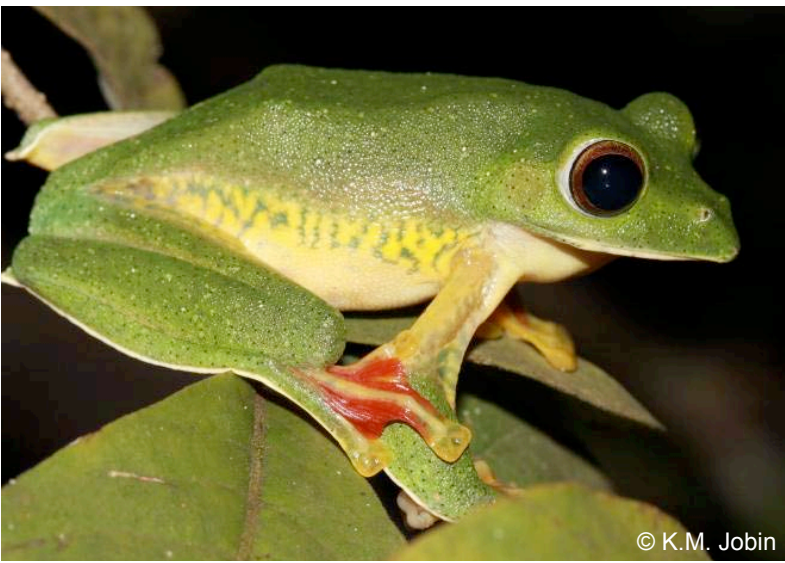

Image 9, Rhacophorus malabaricus Jerdon, 1870

the arm and on hind limb from tarsus to tip of fourth toe (Image 10).

Two calling males were sighted from bushes and among the ground ferns near an artificial water hole between the evergreen forest and tea plantation at Shekkalmudi, PKTR at an altitude of $1118 \mathrm{~m}$. The type locality of this species is Andiparai Shola, Indira Gandhi Wildlife Sanctuary, Tamil Nadu (Vasudevan \& Dutta 2000), and until now it is known only from the type locality. The present observation thus is a new site record for the R. pseudomalabaricus. However, both these location are within the Anamalai hills of the Western Ghats. It is also interesting to note that $R$. pseudomalabaricus and $R$. calcadensis were found in the same pond at Shekkalmudi in PKTR. According to IUCN criteria B1ab(iii)ver 3.1. R. pseudomalabaricus is Critically Endangered (Biju et al. 2004a).

\section{Conclusion}

The present study shows the significance of the Parambikulam Tiger Reserve on the conservation of amphibians particularly the rhacophorids . Out of the 52 species of rhacophorids in Western Ghats, 11 (21.15\%) have been recorded from PKTR during the present study. Many of these sightings of the rhacophorids are of significance. For example, the sighting of Rhacophorus calcadensis is the first record of this species outside the Kalakkad-Mundanthurai Tiger Reserve in Tamil Nadu. Raorchestes nerostagona the present sighting is the first record south of Palakkad Gap, for $R$. ponmudi and $R$. anili, this is the first record from the Anamalai Hills. Raorchestes marki, R. jayarami and Rhacophorus pseudomalabaricus are first records from PKTR.

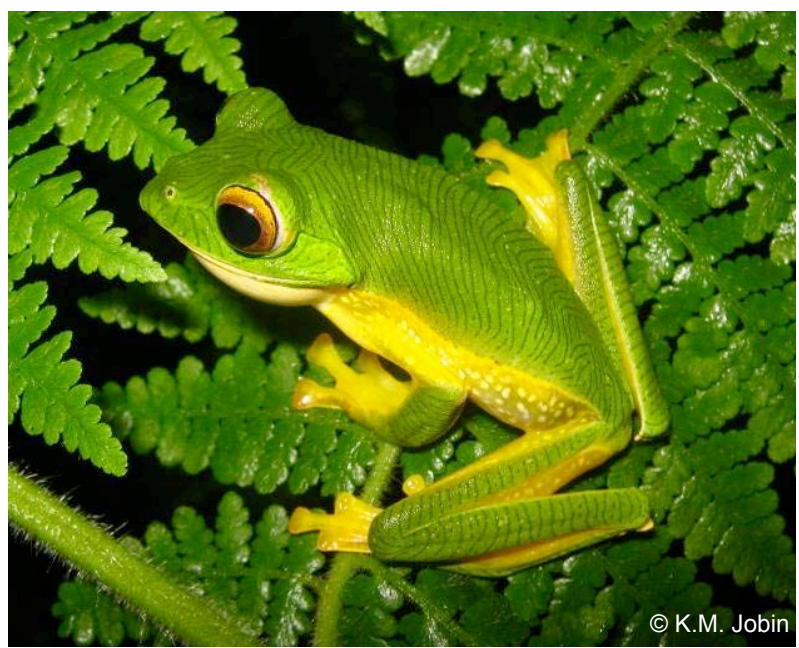

Image 10, Rhacophorus pseudomalabaricus Vasudevan \& Dutta, 2000

Of the 11 species of rhacophorids three are Critically Endangered (CR), two Endangered (EN), and one Data Deficient (DD). Thus nearly $50 \%$ of the rhacophorids of PKTR are threatened with extinction. But if a reassessment of conservation status of these species is done using the additional distributional information, the status would change considerably. It thus warrants immediate attention of the reserve managers on the conservation of the amphibians in their management prescriptions. It also underlines the necessity to measure the wealth of amphibians in the Western Ghats in general and Kerala in particular, as many of the rhacophorids included in this paper are the first report of those species from PKTR. If detailed explorations are done in Parambikulam and other parts of the Western Ghats, the results would be rewarding. Moreover, the result of the study also show how important is it to protect every bit of the biodiversity rich Western Ghats.

\section{REFERENCE}

Biju, S.D. (2003). Reproductive mode in the Shrub Frog Philautus glandulosus (Jerdon, 1853) (Anura: Rhacophoridae). Current Science 84(3): 283-284.

Biju, S.D. (2004). Raorchestes ponmudi. In: IUCN 2011. IUCN Red List of Threatened Species. Version 2011.2. <www. iucnredlist.org> Downloaded on 14 March 2012.

Biju, S.D. (2006). Raorchestes anili. In: IUCN 2011. IUCN Red List of Threatened Species. Version 2011.2. <www. iucnredlist.org>. Downloaded on 14 March 2012.

Biju, S.D. \& F. Bossuyt (2005a). A new species of frog 


\begin{tabular}{|c|c|c|c|c|c|c|c|c|c|c|c|c|c|c|c|c|c|c|c|c|c|c|c|c|}
\hline 운 & $\underset{\dot{\rho}}{\stackrel{\Sigma}{0}}$ & $\mid \begin{array}{l}\infty \\
\stackrel{\infty}{0} \\
\stackrel{0}{0}\end{array}$ & $\mid \begin{array}{l}\infty \\
\stackrel{\infty}{0} \\
\stackrel{0}{0}\end{array}$ & $\mid \begin{array}{l}9 \\
\dot{0} \\
0\end{array}$ & $\stackrel{\hat{\sigma}}{0}$ & $\stackrel{\sim}{\stackrel{N}{N}}$ & $\stackrel{\sim}{\sim}$ & $\stackrel{\stackrel{\leftrightarrow}{\sim}}{\sim}$ & $\stackrel{9}{\stackrel{1}{i}}$ & $\begin{array}{l}\hat{0} \\
0\end{array}$ & $\stackrel{\infty}{\sigma}$ & \begin{tabular}{|l}
$\bar{N}$ \\
$\stackrel{N}{\sigma}$
\end{tabular} & $\begin{array}{l}\vec{J} \\
\stackrel{j}{\circ}\end{array}$ & 苞 & 号 & $\begin{array}{l}\stackrel{R}{\wedge} \\
\wedge\end{array}$ & 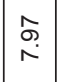 & $\underset{\infty}{\infty}$ & 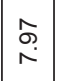 & $\frac{\infty}{0}$ & $\stackrel{\stackrel{f}{F}}{\stackrel{F}{F}}$ & $\mid \begin{array}{l}\bar{\alpha} \\
\infty\end{array}$ & $\stackrel{\text { s. }}{r}$ & 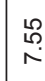 \\
\hline 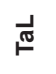 & $\begin{array}{l}\hat{0} \\
i\end{array}$ & $\underset{\substack{n \\
\infty}}{\bar{n}}$ & $\mid$\begin{tabular}{l|}
$\infty$ \\
$q$ \\
$\infty$
\end{tabular} & $\underset{\infty}{\sim}$ & $\begin{array}{l}- \\
0 \\
0\end{array}$ & $\begin{array}{l}\stackrel{R}{R} \\
6\end{array}$ & $\begin{array}{l}\mathscr{8} \\
\dddot{0} \\
\circlearrowleft\end{array}$ & $\begin{array}{l}\stackrel{0}{\wedge} \\
\wedge\end{array}$ & $\begin{array}{l}8 \\
0 \\
6\end{array}$ & $\stackrel{0}{0}$ & $\hat{\imath}$ & $\begin{array}{l}0 \\
\infty\end{array}$ & $\mid \begin{array}{l}8 \\
\infty \\
\infty\end{array}$ & 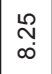 & $\mid \begin{array}{l}\infty \\
\stackrel{0}{0} \\
0\end{array}$ & $\mid \begin{array}{l}\hat{N} \\
\dot{\theta}\end{array}$ & $\stackrel{\circ}{r}$ & $\stackrel{m}{i}$ & $\stackrel{f}{\stackrel{1}{i}}$ & $\begin{array}{l}\text { ปै } \\
0\end{array}$ & $\mid \begin{array}{l}\bar{\sigma} \\
\sigma\end{array}$ & \begin{tabular}{|l|l}
$\infty$ \\
$\infty$ \\
0
\end{tabular} & 艿 & $\begin{array}{l}\stackrel{8}{0} \\
0\end{array}$ \\
\hline$\frac{\vec{b}}{\omega}$ & 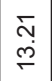 & $\mid \begin{array}{l}\mathscr{O} \\
\stackrel{\rho}{\rho}\end{array}$ & 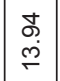 & 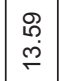 & $\begin{array}{l}\hat{m} \\
0\end{array}$ & $\stackrel{+}{\stackrel{\circ}{\circ}}$ & \begin{tabular}{l}
0 \\
$\stackrel{0}{0}$ \\
\hdashline
\end{tabular} & $\mid$\begin{tabular}{l}
0 \\
$\vdots$ \\
\hdashline
\end{tabular} & 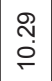 & $\begin{array}{c}\tilde{j} \\
\dot{0}\end{array}$ & $\begin{array}{l}\stackrel{\mathscr{D}}{\sim} \\
\stackrel{\sim}{\sim}\end{array}$ & $\mid \begin{array}{c}\stackrel{N}{\sim} \\
\stackrel{N}{N}\end{array}$ & 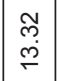 & $\begin{array}{l}\vec{d} \\
\stackrel{\sim}{\sim}\end{array}$ & 足 & 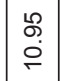 & $\begin{array}{l}\bar{N} \\
\stackrel{\Gamma}{\Gamma}\end{array}$ & $\underset{\underset{N}{F}}{F}$ & $\stackrel{m}{\stackrel{m}{\Gamma}}$ & $\stackrel{n}{0}$ & $\mid \begin{array}{l}\frac{\theta}{\dot{D}} \\
\stackrel{\varphi}{\sim}\end{array}$ & $\underset{\mid \stackrel{+}{F}}{\stackrel{\Delta}{F}}$ & $\underset{\substack{\infty \\
\stackrel{\infty}{\circ}}}{ }$ & \begin{tabular}{l}
8 \\
0 \\
\hdashline
\end{tabular} \\
\hline$\vec{r}$ & $\begin{array}{l}\overline{\grave{j}} \\
\stackrel{\sim}{\mathrm{v}}\end{array}$ & 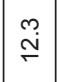 & $\begin{array}{l}\stackrel{+}{\mathrm{j}} \\
\stackrel{\sim}{\mathrm{j}}\end{array}$ & $\begin{array}{l}\stackrel{N}{\mathrm{~N}} \\
\stackrel{\sim}{N}\end{array}$ & \begin{tabular}{|l}
0 \\
$\tilde{o}$ \\
0
\end{tabular} & \& & 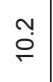 & $\begin{array}{l}\infty \\
\stackrel{0}{\circ}\end{array}$ & $\begin{array}{l}\tilde{N} \\
\stackrel{\rho}{\circ}\end{array}$ & $\begin{array}{l}m \\
\stackrel{9}{0}\end{array}$ & $\stackrel{\stackrel{\infty}{N}}{\stackrel{N}{N}}$ & 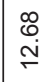 & $\begin{array}{l}\stackrel{\mathcal{U}}{\mathrm{g}} \\
\end{array}$ & 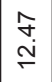 & $\begin{array}{c}\mathbb{N} \\
0\end{array}$ & $\stackrel{\stackrel{m}{F}}{F}$ & 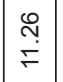 & $\begin{array}{l}\text { f. } \\
\stackrel{0}{0}\end{array}$ & $\begin{array}{c}\dot{\sigma} \\
\stackrel{\Gamma}{\Gamma}\end{array}$ & $\begin{array}{l}\hat{f} \\
0\end{array}$ & $\begin{array}{l}\stackrel{\sim}{ } \\
\stackrel{\omega}{\sim}\end{array}$ & $\bar{O}$ & $\begin{array}{l}\infty \\
\stackrel{0}{0}\end{array}$ & $\begin{array}{l}\stackrel{0}{0} \\
\stackrel{0}{0}\end{array}$ \\
\hline$\frac{\vec{x}}{\mathbf{x}}$ & $\begin{array}{l}\swarrow \\
0 \\
0\end{array}$ & $\mid \begin{array}{l}0 \\
e \\
0\end{array}$ & \begin{tabular}{|l|} 
\\
$\stackrel{\infty}{\infty}$ \\
$\sim$
\end{tabular} & 号 & \begin{tabular}{|c}
$\infty$ \\
$\infty$ \\
0 \\
0
\end{tabular} & $\underset{+}{\mathbb{*}}$ & $\begin{array}{l}\infty \\
œ \\
\dot{\sigma}\end{array}$ & 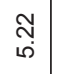 & $\begin{array}{l}\dot{J} \\
\dot{\sigma} \\
\dot{\sigma}\end{array}$ & $\mid \begin{array}{l}0 \\
\tilde{m} \\
0\end{array}$ & \begin{tabular}{|l}
$\infty$ \\
\hdashline \\
$i n$
\end{tabular} & $\begin{array}{l}8 \\
0 \\
0\end{array}$ & $\mid \begin{array}{c}0 \\
\infty \\
0\end{array}$ & 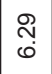 & $\begin{array}{l}0 \\
0 \\
0\end{array}$ & $\mid \begin{array}{l}7 \\
0 \\
6\end{array}$ & \begin{tabular}{|l|}
0 \\
$\vdots$ \\
0
\end{tabular} & 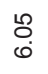 & $\begin{array}{l}\widetilde{\gamma} \\
0\end{array}$ & $\begin{array}{c}\widetilde{N} \\
0\end{array}$ & $\mid \begin{array}{c}7 \\
\infty \\
\infty\end{array}$ & \begin{tabular}{|l}
0 \\
0 \\
$i$
\end{tabular} & $\begin{array}{l}\infty \\
\stackrel{\infty}{\circ}\end{array}$ & $\begin{array}{l}8 \\
0 \\
0\end{array}$ \\
\hline 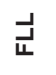 & $\begin{array}{l}\mathscr{L} \\
\infty \\
\wp\end{array}$ & $\begin{array}{l}\infty \\
o \\
0\end{array}$ & $\mid \begin{array}{l}\Re \\
0 \\
0\end{array}$ & $\mid \begin{array}{c}\tilde{c} \\
0 \\
0\end{array}$ & 足 & $\stackrel{\leftrightarrow}{\dot{\tau}}$ & $\bar{i}$ & 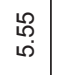 & $\frac{\infty}{i \infty}$ & $\begin{array}{c}\mathscr{0} \\
0 \\
0\end{array}$ & $\begin{array}{l}9 \\
i \\
i\end{array}$ & \begin{tabular}{|c|c}
$\infty$ \\
$\infty$ \\
0 \\
0
\end{tabular} & $\begin{array}{l}\hat{y} \\
\dot{0}\end{array}$ & 文 & 号 & $\begin{array}{l}\stackrel{R}{2} \\
\stackrel{\omega}{\circ}\end{array}$ & \begin{tabular}{|l|}
$\Gamma$ \\
$\hat{i}$
\end{tabular} & య⿵门 & $\mid \begin{array}{l}\bar{\sigma} \\
i\end{array}$ & $\begin{array}{c}\bar{n} \\
0\end{array}$ & 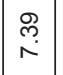 & $\mid \begin{array}{l}\infty \\
i \rho j \\
\llcorner\end{array}$ & $\stackrel{\infty}{\infty}$ & o. \\
\hline$\vec{w}$ & 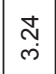 & 导 & $\mid \begin{array}{l}\hat{\omega} \\
\dot{\omega}\end{array}$ & 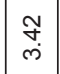 & $\frac{\hat{f}}{0}$ & $\stackrel{\leftrightarrow}{\stackrel{\leftrightarrow}{i}}$ & $\begin{array}{l}\stackrel{0}{\infty} \\
\stackrel{\sim}{\sim}\end{array}$ & $\underset{\sim}{\sim}$ & $\begin{array}{l}\mathfrak{N} \\
\mathfrak{N}\end{array}$ & $\frac{\infty}{0}$ & $\underset{\sim}{\sim}$ & $\begin{array}{l}\hat{\omega} \\
\text { pr }\end{array}$ & $\mid \begin{array}{c}\stackrel{T}{N} \\
\dot{\omega}\end{array}$ & $\begin{array}{l}\stackrel{0}{0} \\
\text { m. }\end{array}$ & $\begin{array}{c}\stackrel{\infty}{1} \\
\stackrel{0}{0}\end{array}$ & \begin{tabular}{|l|}
$\infty$ \\
0 \\
$\dot{\infty}$
\end{tabular} & \begin{tabular}{|c|}
$\stackrel{\sim}{\sim}$ \\
$\stackrel{\sim}{i}$
\end{tabular} & 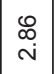 & $\begin{array}{l}\stackrel{\leftrightarrow}{\leftrightarrow} \\
\stackrel{\sim}{~}\end{array}$ & $\underset{0}{F}$ & \begin{tabular}{|l|} 
\\
$\mathscr{m}$ \\
$\dot{m}$
\end{tabular} & $\stackrel{\stackrel{N}{N}}{\stackrel{N}{N}}$ & $\stackrel{\widetilde{N}}{\sim}$ & $\stackrel{\hat{L}}{\mathrm{~N}}$ \\
\hline$\vec{\omega}$ & $\underset{\sim}{\stackrel{P}{+}}$ & 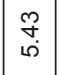 & $\begin{array}{l}\hat{\alpha} \\
\infty \\
\dot{\sigma}\end{array}$ & $\begin{array}{l}\bar{\delta} \\
\dot{\sigma}\end{array}$ & $\begin{array}{l}0 \\
0 \\
0\end{array}$ & ్ָ & $\begin{array}{c}\tilde{N} \\
\text { P. }\end{array}$ & $\begin{array}{l}\stackrel{d}{\oplus} \\
\dot{m}\end{array}$ & $\begin{array}{l}0 \\
0 \\
0 \\
\end{array}$ & $\frac{0}{0}$ & $\begin{array}{l}\stackrel{\rho}{\rho} \\
\sim\end{array}$ & \begin{tabular}{|l}
$\infty$ \\
$\stackrel{\infty}{+}$ \\
$\stackrel{2}{~}$
\end{tabular} & $\begin{array}{l}\stackrel{\leftrightarrow}{\circ} \\
\dot{+}\end{array}$ & 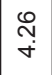 & $\begin{array}{l}0 \\
0 \\
0\end{array}$ & 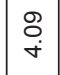 & \begin{tabular}{|l|}
$\infty$ \\
$\infty$ \\
$\infty$
\end{tabular} & $\mid \begin{array}{c}\hat{\rho} \\
\tilde{\rho}\end{array}$ & \begin{tabular}{l|} 
\\
$\infty$ \\
$\dot{m}$
\end{tabular} & $\mid \begin{array}{l}0 \\
\check{N} \\
0\end{array}$ & $\overline{\dot{\theta}}$ & $\begin{array}{l}\stackrel{\leftrightarrow}{ } \\
\stackrel{\sim}{~}\end{array}$ & $\underset{\text { j్ }}{\bar{j}}$ & $\begin{array}{l}\stackrel{g}{9} \\
\text {; }\end{array}$ \\
\hline 嵒 & $\stackrel{N}{\stackrel{N}{\sim}}$ & $\begin{array}{l}\dot{d} \\
\dot{m}\end{array}$ & $\mid \begin{array}{l}0 \\
\stackrel{\leftrightarrow}{\mathrm{N}}\end{array}$ & 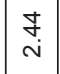 & $\begin{array}{l}\hat{0} \\
0\end{array}$ & $\stackrel{\circ}{r}$ & $\stackrel{\mathcal{F}}{-}$ & $\underset{\stackrel{\leftrightarrow}{\leftarrow}}{\sim}$ & 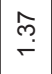 & $\left|\begin{array}{c}\infty \\
\dddot{\infty} \\
0\end{array}\right|$ & $\stackrel{\leftrightarrow}{\stackrel{\leftrightarrow}{+}}$ & 怘 & $\begin{array}{l}\stackrel{g}{a} \\
\text { in }\end{array}$ & $\stackrel{\circ}{i}$ & $\stackrel{\hat{O}}{0}$ & $\stackrel{\text { 导 }}{\leftarrow}$ & مיم & $\stackrel{\check{n}}{-}$ & 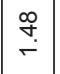 & $\begin{array}{l}0 \\
0 \\
0\end{array}$ & $\underset{i}{\Sigma}$ & $\stackrel{\text { If }}{-}$ & $\stackrel{?}{+}$ & $\underset{+}{\check{-}}$ \\
\hline 岂 & $\begin{array}{l}\Delta \\
\infty \\
\infty\end{array}$ & \begin{tabular}{c}
\multirow{2}{*}{} \\
$\sigma$
\end{tabular} & $\begin{array}{c}\hat{N} \\
\infty\end{array}$ & $\begin{array}{l}\infty \\
\infty \\
\infty \\
\infty\end{array}$ & 离 & $\underset{i s}{i}$ & $\begin{array}{l}\vdots \\
\vdots \\
0\end{array}$ & $\begin{array}{l}\hat{A} \\
\dot{0}\end{array}$ & $\begin{array}{l}\vdots \\
\vdots \\
0\end{array}$ & $\stackrel{m}{0}$ & $\stackrel{\substack{\infty \\
\stackrel{\infty}{\sim}}}{ }$ & $\begin{array}{c}\hat{N} \\
\infty\end{array}$ & $\grave{i}$ & $\stackrel{\mathscr{\Omega}}{\stackrel{\circ}{\circ}}$ & 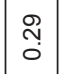 & $\stackrel{i r \rho}{\stackrel{i}{i}}$ & $\stackrel{\infty}{\sim}$ & 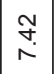 & $\stackrel{?}{\stackrel{P}{\sim}}$ & $\mid \begin{array}{l}\infty \\
0 \\
0\end{array}$ & 量 & \begin{tabular}{|l}
0 \\
0 \\
0
\end{tabular} & $\begin{array}{l}\bar{f} \\
\dot{\theta}\end{array}$ & 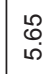 \\
\hline 录 & $\underset{\sigma}{\sigma}$ & $\mid \begin{array}{c}\frac{9}{\dot{O}} \\
\stackrel{0}{1}\end{array}$ & $\begin{array}{l}\infty \\
\infty \\
\infty \\
\infty\end{array}$ & $\begin{array}{l}n \\
\\
\sigma\end{array}$ & $\begin{array}{l}0 \\
0 \\
0 \\
0\end{array}$ & $\stackrel{\bar{N}}{N}$ & $\begin{array}{l}\stackrel{\sim}{\sim} \\
\stackrel{n}{\sim}\end{array}$ & 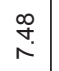 & 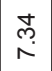 & $\frac{7}{0}$ & $\stackrel{\circ}{\circ}$ & 㝘 & 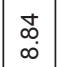 & 占 & $\begin{array}{l}\infty \\
0 \\
0\end{array}$ & $\underset{\substack{\infty\\
}}{\bar{N}}$ & $\begin{array}{l}\infty \\
\stackrel{\infty}{\sim}\end{array}$ & $\underset{\sim}{\stackrel{n}{N}}$ & $\underset{\dot{\sigma}}{\wedge}$ & 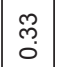 & 惢 & $\mid \begin{array}{l}\tilde{S} \\
0 \\
0\end{array}$ & $\begin{array}{l}\overline{6} \\
0 \\
0\end{array}$ & $\underset{\sim}{\Lambda}$ \\
\hline $\overrightarrow{\underline{I}}$ & $\begin{array}{l}n \\
\hat{\sigma}\end{array}$ & $\begin{array}{l}\mathbb{N} \\
\stackrel{O}{O}\end{array} \mid$ & $\begin{array}{l}\mathbb{N} \\
\sigma\end{array}$ & $\mid \begin{array}{l}\infty \\
\infty \\
\infty\end{array}$ & $\begin{array}{l}0 \\
\stackrel{2}{0} \\
0\end{array}$ & 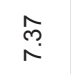 & $\begin{array}{l}\stackrel{0}{\sim} \\
\stackrel{1}{\sim}\end{array}$ & $\begin{array}{l}\stackrel{\leftrightarrow}{\stackrel{\leftrightarrow}{\sim}} \\
\stackrel{0}{*}\end{array}$ & 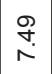 & $\frac{7}{0}$ & $\frac{+}{\sigma}$ & $\begin{array}{l}0 \\
\text { Oे } \\
\text { o. }\end{array}$ & $\begin{array}{l}\infty \\
\infty \\
\infty \\
\infty\end{array}$ & $\begin{array}{l}\text { Jे } \\
\text { o. }\end{array}$ & $\begin{array}{l}f \\
\dot{0}\end{array}$ & $\begin{array}{l}0 \\
0 \\
\infty\end{array}$ & $\begin{array}{l}\dot{O} \\
\dot{\sigma}\end{array}$ & $\stackrel{\leftrightarrow}{\sigma}$ & $\frac{10}{\sigma}$ & $\begin{array}{l}n \\
\\
0\end{array}$ & $\simeq$ & 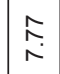 & 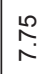 & $\stackrel{+}{\stackrel{t}{\Lambda}}$ \\
\hline क & $\begin{array}{l}\bar{b} \\
\stackrel{N}{ }\end{array}$ & $\frac{\infty}{\dot{N}}$ & $\begin{array}{c}0 \\
2 \\
\stackrel{\rho}{N}\end{array}$ & $\mid \begin{array}{l}\tilde{N} \\
\stackrel{N}{N}\end{array}$ & $\stackrel{\Gamma}{\stackrel{1}{r}}$ & $\stackrel{\infty}{\stackrel{\infty}{\sim}}$ & $\begin{array}{l}\stackrel{\sim}{\sim} \\
\stackrel{N}{N}\end{array}$ & $\begin{array}{l}\hat{\sigma} \\
\grave{\sim}\end{array}$ & $\mid \begin{array}{l}\dot{q} \\
\stackrel{\sim}{N}\end{array}$ & $\mid \begin{array}{l}0 \\
f \\
0\end{array}$ & $\mid$\begin{tabular}{|}
$\stackrel{N}{\sim}$ \\
N
\end{tabular} & 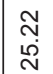 & $\mid \begin{array}{l}\hat{\sigma} \\
\dot{\sim} \\
\text { a }\end{array}$ & $\begin{array}{l}\bar{\infty} \\
\stackrel{+}{\sim}\end{array}$ & $\begin{array}{l}\bar{i} \\
0\end{array}$ & $\begin{array}{l}\mathscr{D} \\
\tilde{N} \\
\text { N }\end{array}$ & $\stackrel{m}{i}$ & 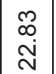 & $\mid \begin{array}{l}\hat{\mathrm{N}} \\
\mathbf{\mathrm { j }}\end{array}$ & $\mid \begin{array}{l}\stackrel{2}{2} \\
0\end{array}$ & $\begin{array}{l}\bar{j} \\
\dot{\rho}\end{array}$ & $\begin{array}{l}\hat{a} \\
\dot{\sim} \\
\dot{N}\end{array}$ & $\begin{array}{l}\infty \\
\stackrel{\infty}{0} \\
\text { in }\end{array}$ & $\begin{array}{l}8 \\
\stackrel{2}{2}\end{array}$ \\
\hline & & & & 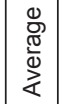 & 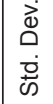 & & & & 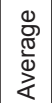 & $\mid \begin{array}{l}\vec{\partial} \\
\text { à } \\
\dot{D} \\
\dot{\omega}\end{array}$ & & & & 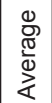 & $\begin{array}{l}\overrightarrow{\dot{\Xi}} \\
\Delta \\
\dot{D} \\
\dot{D}\end{array}$ & & & & 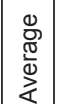 & 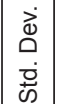 & & & & \\
\hline 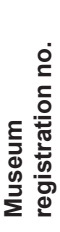 & 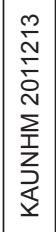 & 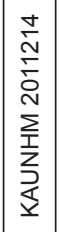 & 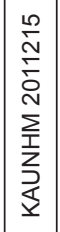 & & & 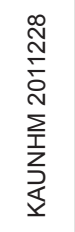 & 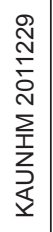 & 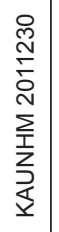 & & & 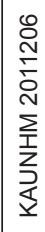 & 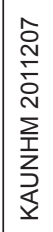 & 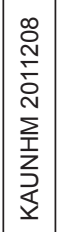 & & & 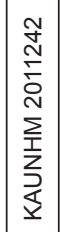 & 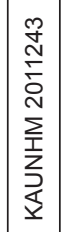 & 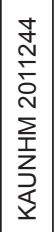 & & & 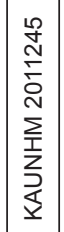 & 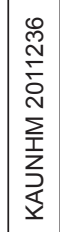 & 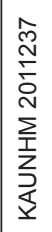 & 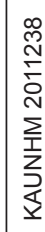 \\
\hline 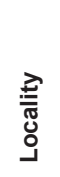 & 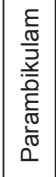 & 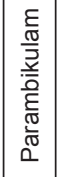 & 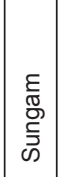 & & & 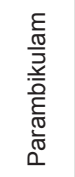 & 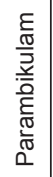 & 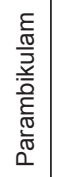 & & & 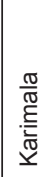 & 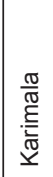 & 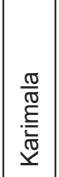 & & & 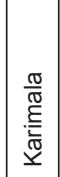 & 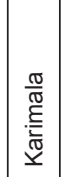 & 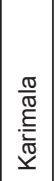 & & & 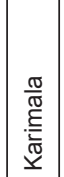 & 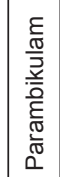 & 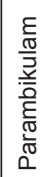 & 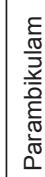 \\
\hline 㖁 & ч & \llcorner & ५ & & & $\Sigma$ & $\Sigma$ & $\Sigma$ & & & $\Sigma$ & $\Sigma$ & $\Sigma$ & & & $\Sigma$ & $\Sigma$ & $\Sigma$ & & & ч & $\Sigma$ & $\Sigma$ & $\Sigma$ \\
\hline $\begin{array}{l}\frac{\mathscr{0}}{0} \\
\text { ळँ } \\
\text { के }\end{array}$ & 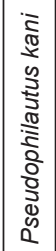 & & & & & 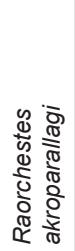 & & & & & 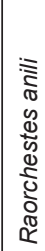 & & & & & 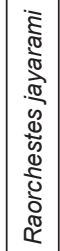 & & & & & & 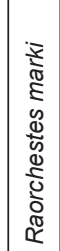 & & \\
\hline
\end{tabular}




\begin{tabular}{|c|c|c|c|c|c|c|c|c|c|c|c|c|c|c|c|c|c|c|}
\hline $\overrightarrow{0}$ & \begin{tabular}{|c|c}
$\infty$ \\
$\stackrel{\infty}{\sim}$ \\
$\wedge$
\end{tabular} & 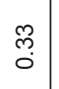 & 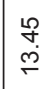 & \begin{tabular}{|l}
$\infty$ \\
$\infty$ \\
$\stackrel{m}{\infty}$
\end{tabular} & \begin{tabular}{|l|}
$\infty$ \\
$\stackrel{\infty}{\leftrightarrow \rho}$ \\
$\stackrel{+}{+}$
\end{tabular} & $\begin{array}{l}\infty \\
\stackrel{\rho}{\omega} \\
\stackrel{\rho}{~}\end{array}$ & $\mid \begin{array}{c}\infty \\
\substack{0 \\
0 \\
0}\end{array}$ & 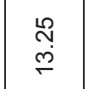 & \begin{tabular}{|}
$\stackrel{\mathscr{P}}{\rightleftarrows}$ \\
$\stackrel{F}{F}$
\end{tabular} & $\stackrel{\stackrel{m}{\rightleftharpoons}}{\stackrel{m}{F}}$ & $\simeq$ & 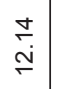 & $\left|\begin{array}{l}\bar{\infty} \\
\stackrel{\infty}{0}\end{array}\right|$ & & $\stackrel{\sim}{\stackrel{\sim}{\sim}}$ & $\begin{array}{l}\stackrel{\jmath}{\infty} \\
\stackrel{\infty}{\leftarrow}\end{array}$ & 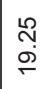 & 䓌 \\
\hline 元 & \begin{tabular}{|l|}
$\infty$ \\
0 \\
0 \\
0
\end{tabular} & $\bar{m}$ & \begin{tabular}{|l}
$\infty$ \\
$\stackrel{0}{0}$ \\
$\stackrel{0}{0}$
\end{tabular} & 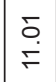 & $\begin{array}{l}\stackrel{N}{\stackrel{N}{\Gamma}} \\
\stackrel{5}{*}\end{array}$ & \begin{tabular}{l|}
\multirow{2}{*}{} \\
$\stackrel{\circ}{\circ}$
\end{tabular} & $\begin{array}{c}\infty \\
\infty \\
0 \\
0\end{array}$ & ুু & \begin{tabular}{|l|}
$\bar{N}$ \\
$\stackrel{N}{\circ}$
\end{tabular} & $\stackrel{\infty}{\infty}$ & $\stackrel{\stackrel{0}{0}}{\circ}$ & $\begin{array}{l}\tilde{O} \\
\stackrel{0}{\circ}\end{array}$ & ֶ̊: & 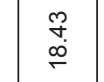 & $\stackrel{\stackrel{g}{+}}{\stackrel{f}{\leftarrow}}$ & 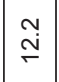 & $\begin{array}{l}\stackrel{\infty}{\infty} \\
\stackrel{\rho}{\rho}\end{array}$ & 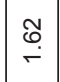 \\
\hline $\overrightarrow{\vec{c}}$ & $\begin{array}{l}\stackrel{0}{0} \\
\infty \\
0 \\
0\end{array}$ & $\stackrel{\stackrel{\sim}{N}}{0}$ & 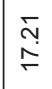 & 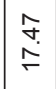 & \begin{tabular}{|l|}
$\infty$ \\
$\infty$ \\
$\infty$ \\
$\infty$
\end{tabular} & \begin{tabular}{|l|}
0 \\
$\infty$ \\
$\stackrel{\sim}{\sim}$
\end{tabular} & 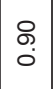 & $\begin{array}{l}\stackrel{m}{\infty} \\
\stackrel{\leftrightarrow}{\leftarrow}\end{array}$ & $\mid \begin{array}{l}\tilde{\alpha} \\
\dot{6} \\
-\end{array}$ & $\begin{array}{l}\mathscr{P} \\
\stackrel{+}{+}\end{array}$ & $\stackrel{m}{\stackrel{m}{\Gamma}}$ & $\begin{array}{l}\stackrel{8}{0} \\
\dot{6}\end{array}$ & $\stackrel{\stackrel{\leftrightarrow}{\circ}}{\circ}$ & $\begin{array}{l}\stackrel{\circ}{\circ} \\
\dot{m}\end{array}$ & 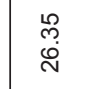 & $\begin{array}{l}\hat{N} \\
\tilde{N}\end{array}$ & $\begin{array}{l}\bar{\infty} \\
\stackrel{+}{\sim}\end{array}$ & $\stackrel{\infty}{\stackrel{i}{i}}$ \\
\hline$\vec{f}$ & \begin{tabular}{|l|}
$\infty$ \\
0 \\
0 \\
0
\end{tabular} & 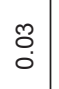 & $\begin{array}{l}0 \\
0 \\
0 \\
0\end{array}$ & 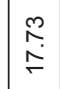 & \begin{tabular}{|l|}
$\infty$ \\
$\infty$ \\
$\infty$ \\
$\leftarrow$
\end{tabular} & $\begin{array}{c}\stackrel{\infty}{\infty} \\
\stackrel{\sim}{\sim}\end{array}$ & $\underset{\dot{r}}{-}$ & $\begin{array}{l}\stackrel{\circ}{\circ} \\
\dot{+}\end{array}$ & $\begin{array}{l}\stackrel{\sim}{\sim} \\
\stackrel{2}{*}\end{array}$ & $\begin{array}{l}\stackrel{m}{0} \\
\stackrel{\leftrightarrow}{\circ}\end{array}$ & $\begin{array}{l}\stackrel{\rho}{e} \\
\stackrel{6}{\sigma}\end{array}$ & $\begin{array}{l}\hat{a} \\
\dot{\rho} \\
\stackrel{b}{\circ}\end{array}$ & $\underset{\stackrel{\sigma}{r}}{\stackrel{\delta}{*}}$ & $\begin{array}{l}\stackrel{+}{\infty} \\
\stackrel{\infty}{\sim}\end{array}$ & $\stackrel{\circ}{\stackrel{0}{N}}$ & 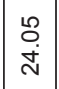 & $\begin{array}{l}\stackrel{m}{\sim} \\
\stackrel{d}{\sim}\end{array}$ & 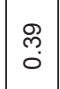 \\
\hline$\underset{\mathbb{x}}{\vec{x}}$ & $\begin{array}{l}2 \\
\stackrel{2}{i n} \\
i \infty\end{array}$ & $\begin{array}{c}\text { ల్ } \\
\text { O }\end{array}$ & 号 & 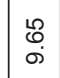 & $\mid$\begin{tabular}{l}
0 \\
0 \\
\hdashline \\
\hdashline
\end{tabular} & $\begin{array}{l}\infty \\
\stackrel{\infty}{\infty} \\
\sigma\end{array}$ & $\mid \begin{array}{c}\stackrel{0}{0} \\
\infty \\
0\end{array}$ & 㲾 & \begin{tabular}{l|}
$\bar{\infty}$ \\
$\infty$ \\
$\infty$
\end{tabular} & 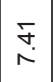 & 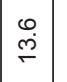 & $\stackrel{m}{\sigma}$ & 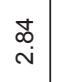 & 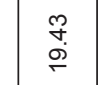 & $\begin{array}{l}\stackrel{0}{\stackrel{\sim}{N}} \\
\stackrel{\sim}{\sim}\end{array}$ & $\mid \stackrel{\underset{N}{\mathrm{~N}}}{\sim}$ & 竎 & 骂 \\
\hline મ્ & \begin{tabular}{l|}
$\stackrel{9}{2}$ \\
$i \infty$
\end{tabular} & $\begin{array}{c}\stackrel{\bullet}{\infty} \\
0 \\
0\end{array}$ & 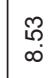 & $\begin{array}{l}\hat{m} \\
\dot{\sigma}\end{array}$ & $\begin{array}{l}\Sigma \\
\dot{\sigma}\end{array}$ & 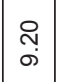 & $\begin{array}{l}\overline{0} \\
0\end{array}$ & مo & 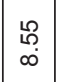 & 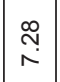 & 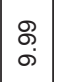 & $\underset{\infty}{\mathbb{\infty}}$ & $\stackrel{\stackrel{m}{\leftrightarrow}}{\stackrel{2}{*}}$ & 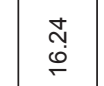 & 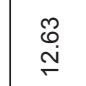 & $\mid \begin{array}{l}\hat{\stackrel{f}{*}} \\
\stackrel{+}{F}\end{array}$ & \begin{tabular}{|l}
$\stackrel{\leftrightarrow}{\infty}$ \\
$\stackrel{\infty}{\rightleftharpoons}$
\end{tabular} & $\stackrel{\circ}{\stackrel{\circ}{\longrightarrow}}$ \\
\hline$\vec{\Psi}$ & 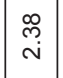 & $\stackrel{\widetilde{N}}{0}$ & $\underset{\dot{F}}{\mathcal{F}}$ & 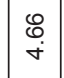 & $\underset{\dot{F}}{\check{f}}$ & $\underset{f}{\check{f}}$ & $\stackrel{\stackrel{n}{N}}{\stackrel{N}{0}}$ & $\underset{\sim}{\stackrel{R}{\rho}}$ & $\stackrel{\stackrel{2}{\mid}}{\underset{f}{+}}$ & $\begin{array}{l}\hat{D} \\
\dot{m}\end{array}$ & 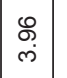 & $\stackrel{\hat{\sigma}}{\dot{m}}$ & $\stackrel{\circ}{\stackrel{0}{0}}$ & $\stackrel{\infty}{6}$ & $\underset{i \infty}{\infty}$ & $\begin{array}{l}\infty \\
i \infty \\
\infty\end{array}$ & \begin{tabular}{|c|}
$\infty$ \\
if \\
if
\end{tabular} & $\begin{array}{l}0 \\
0 \\
0\end{array}$ \\
\hline$\vec{\omega}$ & $\begin{array}{l}\underset{\sim}{\tilde{j}} \\
\dot{n}\end{array}$ & $\hat{m}$ & 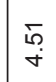 & 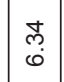 & $\mid$\begin{tabular}{l|}
$\hat{\infty}$ \\
$i$ \\
$i$
\end{tabular} & $\begin{array}{l}\hat{\omega} \\
\dot{\omega}\end{array}$ & $\begin{array}{l}\stackrel{\leftrightarrow}{o} \\
\stackrel{0}{0}\end{array}$ & $\underset{\infty}{\widetilde{\infty}}$ & $\begin{array}{l}\stackrel{\tilde{e}}{0} \\
\dot{0}\end{array}$ & $\frac{\cong}{\stackrel{0}{0}}$ & $\overline{0}$ & $\begin{array}{l}\tilde{\lambda} \\
\dot{0}\end{array}$ & $\stackrel{\sim}{\stackrel{\sigma}{\sigma}}$ & 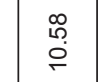 & $\underset{\infty}{\stackrel{N}{\sim}}$ & $\underset{\stackrel{\mathcal{F}}{\stackrel{2}{r}}}{ }$ & 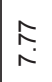 & 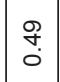 \\
\hline 盝 & 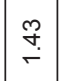 & 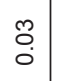 & $\stackrel{\mathcal{N}}{\mathrm{N}}$ & 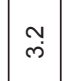 & $\frac{\stackrel{\rho}{r}}{\dot{m}}$ & $\frac{\circ}{\dot{m}}$ & $\stackrel{\uparrow}{0}$ & $\stackrel{\leftrightarrow}{i}$ & $\stackrel{户}{~}$ & $\begin{array}{l}\stackrel{\infty}{\infty} \\
\stackrel{\infty}{\sim}\end{array}$ & 灾 & $\begin{array}{c}\infty \\
\stackrel{\infty}{\infty} \\
\sim\end{array}$ & $\begin{array}{l}\stackrel{0}{0} \\
0\end{array}$ & స్త్ర & 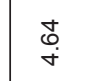 & $\begin{array}{l}\stackrel{9}{2} \\
\dot{m}\end{array}$ & 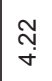 & $\begin{array}{l}0 \\
\vdots \\
0\end{array}$ \\
\hline$\underline{\underline{\underline{u}}}$ & $\begin{array}{l}\dot{d} \\
0 \\
0\end{array}$ & 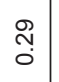 & $\underset{\Gamma}{\stackrel{\Gamma}{\Gamma}}$ & $\underset{⿱ 乛}{\stackrel{F}{F}}$ & \begin{tabular}{|c|}
$\stackrel{\tilde{N}}{\Gamma}$ \\
$\stackrel{\Gamma}{\Gamma}$
\end{tabular} & 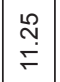 & $\begin{array}{l}\mathscr{m} \\
0 \\
0\end{array}$ & $\stackrel{\hat{m}}{\stackrel{\rho}{\rho}}$ & $\frac{N}{\infty}$ & 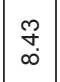 & $\begin{array}{c}\bar{N} \\
\infty\end{array}$ & $\begin{array}{l}\infty \\
\substack{\infty \\
\infty}\end{array}$ & $\stackrel{\hat{o}}{\dot{r}}$ & 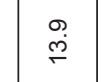 & $\begin{array}{l}\frac{8}{d} \\
\stackrel{d}{\leftarrow}\end{array}$ & \begin{tabular}{|l|}
$\stackrel{\mathscr{p}}{\rho}$ \\
$\stackrel{\rho}{\rho}$
\end{tabular} & $\begin{array}{l}\stackrel{g}{q} \\
\dot{j}\end{array}$ & 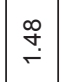 \\
\hline 3 & \begin{tabular}{|l|} 
\\
0 \\
0
\end{tabular} & 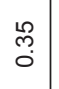 & $\stackrel{\circ}{\circ}$ & 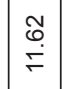 & $\mid \begin{array}{l}\hat{\oplus} \\
\stackrel{\Gamma}{F}\end{array}$ & $\stackrel{\stackrel{P}{P}}{\stackrel{P}{F}}$ & 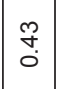 & $\stackrel{\stackrel{\leftrightarrow}{+}}{\stackrel{+}{+}}$ & $\mid \begin{array}{c}\underset{\mathfrak{d}}{\mathrm{I}} \\
\end{array}$ & $\begin{array}{l}\stackrel{\infty}{\infty} \\
\stackrel{\leftrightarrow}{\rightleftharpoons} \\
\end{array}$ & 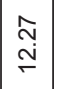 & $\begin{array}{c}\stackrel{\infty}{\stackrel{\infty}{\Gamma}} \\
\stackrel{\Gamma}{*}\end{array}$ & \begin{tabular}{l}
$\circledast$ \\
\hdashline \\
\hdashline
\end{tabular} & $\begin{array}{l}\infty \\
\infty \\
\stackrel{\infty}{\leftarrow}\end{array}$ & 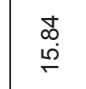 & $\begin{array}{l}\bar{\infty} \\
\dot{+}\end{array}$ & $\begin{array}{l}\infty \\
\tilde{m} \\
\stackrel{\omega}{\omega}\end{array}$ & $\begin{array}{l}\tilde{R} \\
\stackrel{0}{0} \\
0\end{array}$ \\
\hline $\overrightarrow{\underline{x}}$ & $\begin{array}{l}\stackrel{R}{\stackrel{R}{\sim}} \\
\end{array}$ & 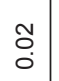 & 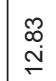 & \begin{tabular}{|l|}
$\stackrel{\circ}{0}$ \\
$\ddot{m}$
\end{tabular} & 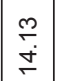 & \begin{tabular}{|l|} 
\\
$\dot{\rho}$ \\
$\stackrel{\rho}{p}$
\end{tabular} & $\stackrel{8}{\circ}$ & $\stackrel{\text { qu}}{\stackrel{9}{0}}$ & \begin{tabular}{|l|}
$\stackrel{O}{F}$ \\
\end{tabular} & \begin{tabular}{|l|}
0 \\
$\infty$ \\
$\stackrel{0}{0}$
\end{tabular} & \begin{tabular}{|l}
$\stackrel{\sim}{\sim}$ \\
$\stackrel{F}{F}$
\end{tabular} & 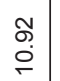 & $\hat{\tilde{m}}$ & $\begin{array}{l}\bar{\circ} \\
\stackrel{\text { ஸे }}{ }\end{array}$ & $\begin{array}{l}\stackrel{o}{0} \\
\stackrel{0}{0}\end{array}$ & 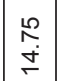 & 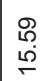 & 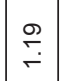 \\
\hline 宑 & 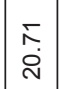 & $\begin{array}{l}8 \\
0 \\
0\end{array}$ & 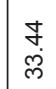 & $\begin{array}{l}\infty \\
\stackrel{\infty}{\dot{f}} \\
\dot{f}\end{array}$ & \begin{tabular}{|l|}
$\bar{m}$ \\
$\infty$ \\
$\infty$
\end{tabular} & 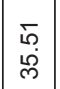 & نָ & 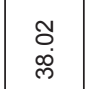 & \begin{tabular}{|l|}
$\mathbb{N}$ \\
$o ̈$ \\
$\dot{m}$
\end{tabular} & \begin{tabular}{|c|}
$\stackrel{\sim}{\tilde{m}}$ \\
$\stackrel{m}{n}$
\end{tabular} & \begin{tabular}{|l|} 
\\
0 \\
0 \\
0
\end{tabular} & $\begin{array}{l}m \\
\stackrel{m}{\dot{m}} \\
\stackrel{m}{\mid}\end{array}$ & ס. & 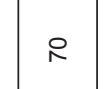 & $\begin{array}{l}\mathscr{8} \\
\infty \\
\dot{\sigma}\end{array}$ & \begin{tabular}{|l|}
$\bar{\delta}$ \\
$\dot{g}$ \\
$\dot{g}$
\end{tabular} & $\begin{array}{l}\mathbf{1} \\
10 \\
0 \\
g\end{array}$ & $\frac{0}{i}$ \\
\hline & 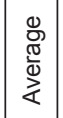 & $\begin{array}{l}\overrightarrow{\dot{D}} \\
\Delta \\
\dot{D} \\
\dot{\omega}\end{array}$ & & & & 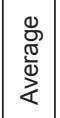 & $\mid \begin{array}{l}\vec{d} \\
\Delta \\
\dot{d} \\
\dot{i} \\
\end{array}$ & & & & & 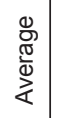 & $\begin{array}{l}\overrightarrow{\dot{D}} \\
\Delta \\
\dot{D} \\
\dot{\omega}\end{array}$ & & & & & \begin{tabular}{|l|}
$\overrightarrow{\dot{D}}$ \\
$\Delta$ \\
$\dot{d}$ \\
$\dot{d}$
\end{tabular} \\
\hline 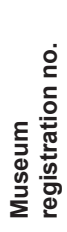 & & & 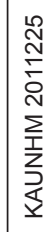 & 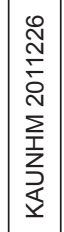 & 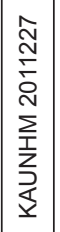 & & & 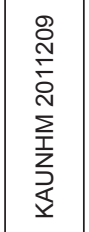 & 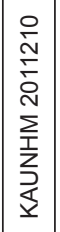 & 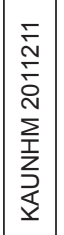 & 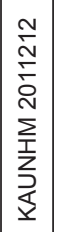 & & & 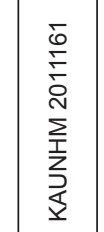 & 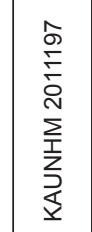 & 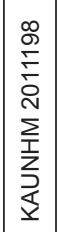 & & \\
\hline 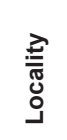 & & & 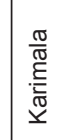 & 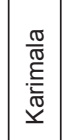 & 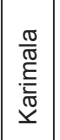 & & & 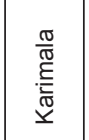 & 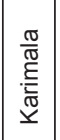 & \begin{tabular}{|l|}
$\frac{\mathbb{N}}{\mathbb{\widetilde { T }}}$ \\
产 \\
$\underline{\mathbb{T}}$ \\
\end{tabular} & 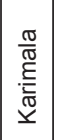 & & & $\begin{array}{l}\frac{\pi}{\pi} \\
\underline{\tilde{E}} \\
\underline{\widetilde{\pi}}\end{array}$ & 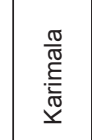 & 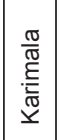 & & \\
\hline$\stackrel{\times}{\varnothing}$ & & & $\Sigma$ & $\Sigma$ & $\Sigma$ & & & $\Sigma$ & $\Sigma$ & $\Sigma$ & $\Sigma$ & & & ч & $\Sigma$ & $\Sigma$ & & \\
\hline$\frac{\mathscr{\Xi}}{\mathscr{\Xi}}$ & & & 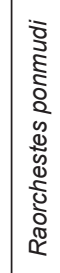 & & & & & 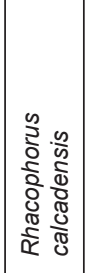 & & & & & & 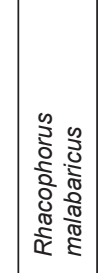 & 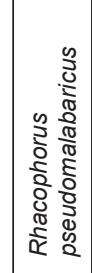 & & & \\
\hline
\end{tabular}


(Ranidae, Rhacophoridae, Philautus) from the rainforest canopy in the Western Ghats, India. Current Science 88: $175-178$.

Biju, S.D. \& F. Bossuyt (2005b). Two new Philautus (Anura, Ranidae, Rhacophorinae) from Ponmudi Hill in the Western Ghats of India. Copeia 1: 29-37.

Biju, S.D. \& F. Bossuyt (2006a). Two new species of Philautus (Anura, Ranidae, Rhacophorinae) from the Western Ghats, India. Amphibia-Reptilia 27: 1-9.

Biju, S.D. \& F. Bossuyt (2006b). Raorchestes nerostagona. In: IUCN 2011. IUCN Red List of Threatened Species. Version 2011.2. <www.iucnredlist.org>. Downloaded on 14 March 2012.

Biju, S.D. \& F. Bossuyt (2009). Systematics and phylogeny of Philautus Gistel, 1848 (Anura, Rhacophoridae) in the Western Ghats of India, with descriptions of 12 new species. Zoological Journal of the Linnaean Society 155: 374-444.

Biju, S.D., Y. Shouche., A. Dubois, S.K. Dutta \& F. Bossuyt (2010). A ground-dwelling rhacophorid frog from the highest mountain peak of the Western Ghats of India. Current Science 98 (8): 119-1125.

Biju, S.D., S.K. Dutta, K. Vasudevan, C. Srinivasulu \& S.P. Vijayakumar (2004a). Rhacophorus pseudomalabaricus. In: IUCN 2011. IUCN Red List of Threatened Species. Version 2011.2. <www.iucnredlist.org>. Downloaded on 14 March 2012.

Biju, S.D., G.D. Bhuddhe, S.K. Dutta, K. Vasudevan, C. Srinivasulu \& S.P. Vijayakumar (2004b). Rhacophorus calcadensis. In: IUCN 2011. IUCN Red List of Threatened Species. Version 2011.2. <www.iucnredlist.org>. Downloaded on 14 March 2012.

Bossuyt, F. (2002). Anew species of Philautus (Anura: Ranidae) from the Western Ghats of India. Journal of Herpetology 36: 656-661.

Bossuyt, F. \& A. Dubois (2001). A review of the frog genus Philautus Gistel, 1848 (Amphibia, Anura, Ranidae, Rhacophorinae), Zeylanica 6(1): 1-112.

Conservation International (2005). Biodiversity Hotspots $<$ www.cnrs.fr/inee/recherche/fichiers/Biodiversite hotspots.pdf $>$. Downloaded on 30 March 2012.

Das, I. (2008). Polypedates occidentalis. In: IUCN 2011. IUCN Red List of Threatened Species. Version 2011.2. <www. iucnredlist.org>. Downloaded on 14 March 2012.

Das, I. \& M.S. Ravichandran (1998). A new species of Polypedates (Anura: Rhacophoridae) from the Western Ghats, India, allied to the Sri Lankan P. cruciger Blyth, 1852. Hamadryad 22: 88-94.

Das, I. \& S.K. Dutta (2006). New species of Polypedates (Anura: Rhacophoridae) from the Western Ghats, southwest India, Journal of Herpetology 40(2): 214-220.

Gururaja, K.V. \& T.V. Ramachandra (2006). Direct development in White-nosed Frog Philautus cf. leucorhinus. Current Science 90(3): 450-454.

Gururaja, K.V., K.P. Dinesh, M.J. Palot, C. Radhakrishnan \& T.V. Ramachandra (2007). A new species of Philautus Gistel (Amphibia: Anura: Rhacophoridae) from southern
Western Ghats, India. Zootaxa 1621: 1-16.

Hegde, V.D. \& G. Bhat (2011). First record of Charpa Tree Frog Polypedates occidentalis from Karnataka State. Frog Leg 15: 10-11.

IUCN SSC Amphibian Specialist Group (2011). Raorchestes marki. In: IUCN 2011. IUCN Red List of Threatened Species. Version 2011.2. <www.iucnredlist.org>. Downloaded on 14 March 2012.

Kaler, O.P. (2011). First Tiger Conservation Plan for Parambikulam Tiger Reserve, 2011-12 to 2020-21. Kerala State Forest and Wildlife Department.

Krishnamurthy, S.V., K.V. Gururaja \& A.H.M. Reddy (2002). Direct development in Philautus glandulosus (Anura: Rhacophoridae). Herpetological Natural History 9(1): 97-102.

Kuramoto, M. \& S.H. Joshy (2003). Two new species of the genus Philautus (Anura: Rhacophoridae) from the Western Ghats, southwestern India. Current Herpetology 22: 5160.

Myers, N., R.A. Mittermeier, C.G. Mittermeier, G.A.B. daFonseca \& J. Kent (2000) Biodiversity hotspots for conservation priorities. Nature 403: 853-858

Patil, N.S. \& R.D. Kanamadi (1997). Direct development in the rhacophorid frog, Philautus variabilis (Gunter). Current Science 73: 697-701.

Purushotham, C.B. \& B. Tapley (2011). Checklist of Amphibians: Agumbe Rainforest Research Station. Frog Leg 16: 2-14.

Radhakrishnan, C. (1996). Amphibians of Parambikulam Wildlife Sanctuary, Kerala. Cobra 26: 10-17.

Rao, C.R.N. (1937). On some new forms of Batrachia from South india. Proceedings of Indian Academy of Sciences. 6(6): 387-427.

Satyamurthi, S.T. (1967). The south Indian amphibian in the collection of the Madras Govt. Museum. Bulletin of Madras Govt. Museum (New Series) (Natural History Section) 7(2): $1-87$.

Vasudevan, K. \& S.K. Dutta (2000). A new species of Rhacophorus (Anura: Rhacophoridae) from the Western Ghats, India. Hamadryad 25(1): 21-28.

Vasudevan, K., A. Kumar \& R. Chellam (2001). Structure and composition of rainforest floor amphibian communities in Kalakad-Mundanthurai Tiger Reserve. Current Science 80(3): 406-412.

Zachariah, A., K.P. Dinesh, E. Kunhikrishnan, S. Das, D.V. Raju, C. Radhakrishnan, M.J. Palot \& S. Kalesh (2011a). Nine new species of frogs of the genus Raorchestes (Amphibia: Anura: Rhacophoridae) from southern Western Ghats, India. Biosystematica 5(1): 24-48.

Zachariah, A., K.P. Dinesh, C. Radhakrishnan, E. Kunhikrishnan, M.J. Palot \& C.K. Vishnudas (2011b). A new species of Polypedates Tschudi (Amphibia: Anura: Rhacophoridae) from southern Western Ghats, Kerala, India. Biosystematica 5(1): 49-53. 